\title{
Digital Transformation in Food Industry- A Consumer Perspective
}

\author{
Ms. Janaki P, Assistant Professor, Surana college, India. janakilokesh1017@gmail.com \\ Prof. Mini.K Abraham, HOD Surana college, India.
}

\begin{abstract}
Digitalisation has created a new revolution in the era of booming businesses, technological advancement and widespread market reach. It has not only affected the educational, hospitality, medical or transportation sectors of the economy but has also acted as a catalyst in the rise of food service industry or fast-food restaurant sectors. The number of fast-food business have substantially increased in the past few years and it is safe to say that digitalisation has played a major role in introducing a new way of food indulgence to consumers, resulting in the increase in businesses pertaining to food. This paper aims to provide a perspective on how digitalisation has affected the food industry from its marketing function to its supply function and how it affects consumers' interest or preferences.
\end{abstract}

Keywords: Advancement, Digitalization, Industry, Service, Technology.

\section{INTRODUCTION}

Digital transformation is the amalgamation of digital technology into all the sectors of a business. The companies that do reconstruct digitally are creating highly engaged and active customers.

India is a young country with almost 60 percent of people in the age window of 18-35 years who are the most receptive group in terms of digitalization. Indian population has now been embracing and exploring the culture of eating out like never before. Since 20th century there has been a massive increase in technology leading to change and transformation in Food Industry and consumer behaviour. Many facets of Food Industry have evolved so as to adopt new technology to meet consumer's expectations and to match their needs which in turn has changed the method of business. Digital technology has also been a major reason for an upsurge in the 'Change in food choice behaviour in Food Industry' and turning it into an easy way of fast paced life for common people at the same time being highly competitive with quick service restaurant.

\section{OBJECTIVES:}

- To understand the effect of digitalisation in changing consumer preferences with regard to fast food industries

- To understand the ripple of booming fast food industries and the role of digitalisation in creating the rise

- To understand the sense of convenience and luxury to consumers created by adoption of digitalisation in eateries and restaurants

- The possible effects of the sudden boom in fast food industry to the economy

\section{RESEARCH METHODOLOGY}

- Primary data collected through survey of 40 consumers, aged between 16-22 years. This survey consisted of 7 multiple choice questions where the participants chose the most appropriate or relatable answers LIMITATIONS:

- Concentrates only on the fast food sector not the complete food industry (manufacturing \& processing function)

- Primary Data collected was limited to students between the ages $16-22$

- Primary Data collected limited to 40 survey participants

Statement: Digitalisation in the fast food industry has affected consumer preferences favourably.

Analysis: A survey was conducted among 40 people with the age range of 16 to 22 years to understand their preferences pertaining to food.

Fig -1

How often do you order food online 40 responses

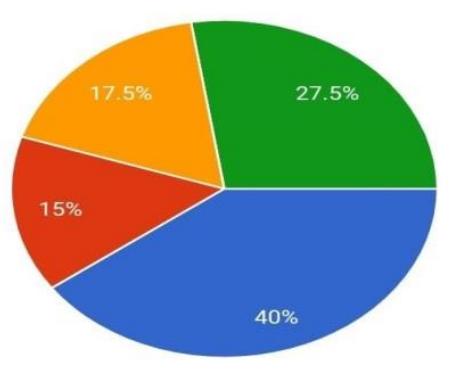

Very often Often Occasionally Rarely 
INFERENCE: We can observe in fig 2 that $40 \%$ of the participants order food online very often, $15 \%$ order often, whereas the rest $45 \%$ responses includes "occasionally" and "rarely" response.

Fig - 2

Are you an active user of swiggy, zomato or any such food delivery apps

40 responses

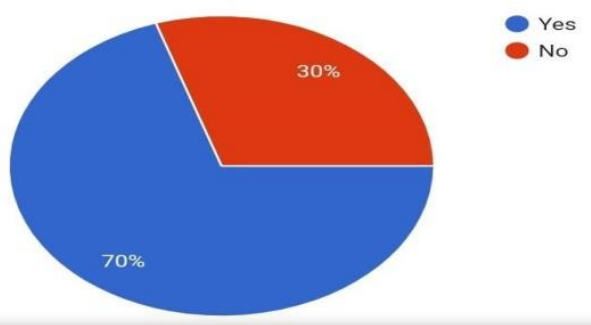

INFERENCE: We can observe in fig. 1 that 28 out of 40 people, i.e. $70 \%$ of the people participated are active users of food delivery apps.

Fig - 3

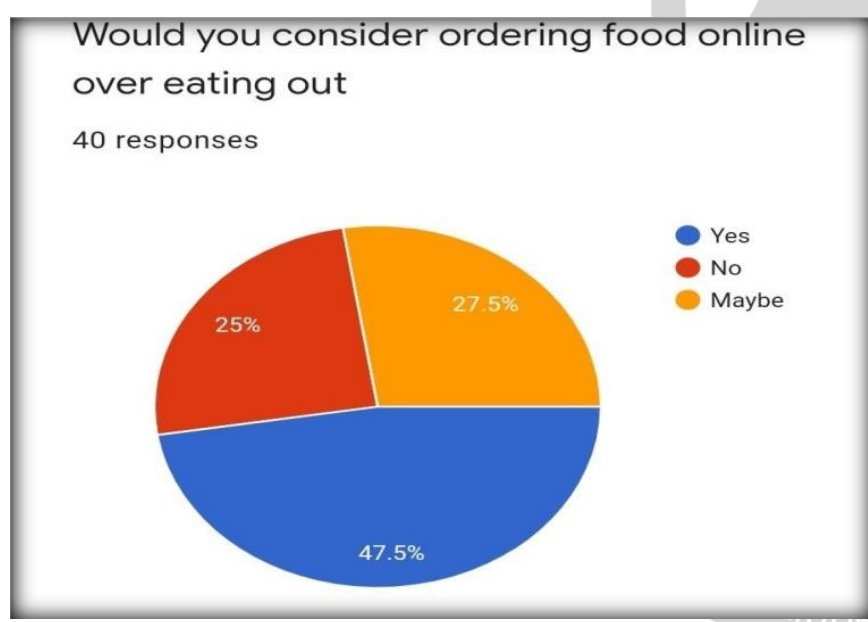

INFERENCE: Fig 3 depicts $47.5 \%$ people prefer ordering online against the $25 \%$ response who chose eating out and $27.5 \%$ unsure response.

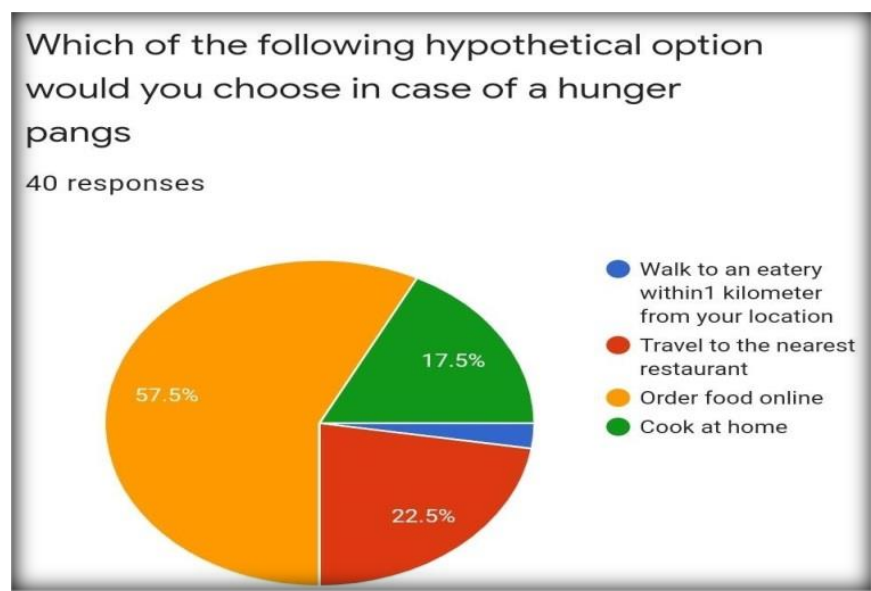

Fig -4
INFERENCE: We can observe in Fig 4 that 23 out of 40 participants i.e. $57.5 \%$ people prefer ordering food online rather than going to an eatery or cook at home.

Do you think it would be easy for you to survive without food delivery apps in this fast paced world

40 responses

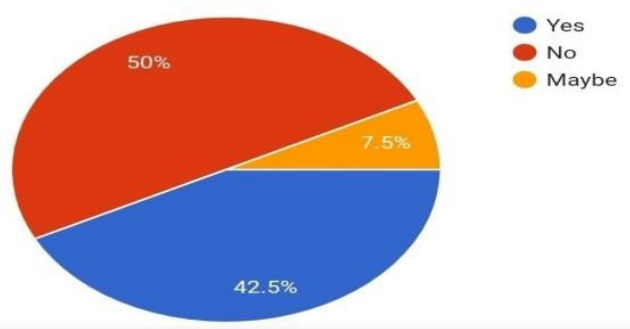

Fig -5

INFERENCE: We can observe in fig 5 that $50 \%$ of the participants admit that they over indulge in their eating habits due to easy ordering and doorstep delivery.

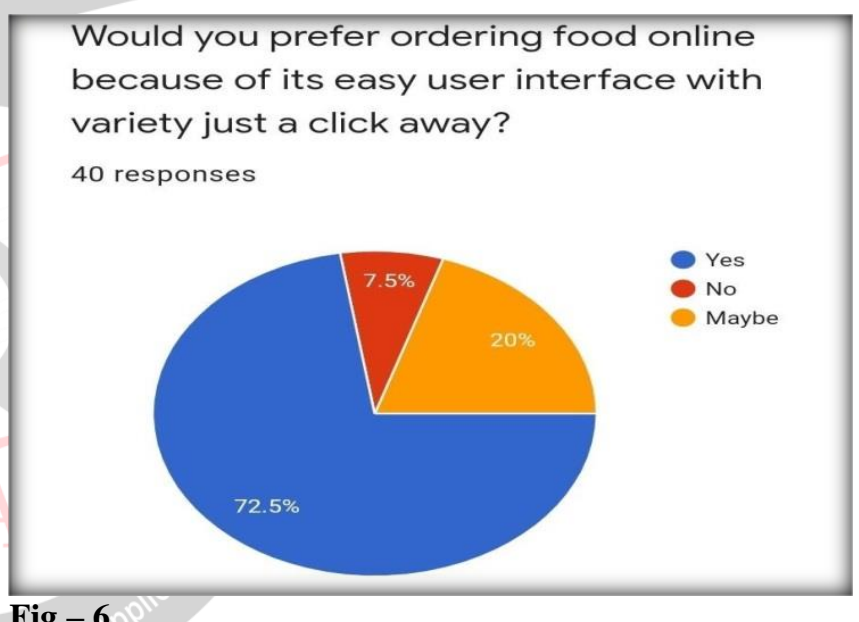

Fig - 6

INFERENCE: By observing Fig 6, we can infer that the digital mode of purchase and easy interface attract $72.5 \%$ participants to choose online food ordering.

Fig -7

So you think you sometimes over indulge in eating because of the easy door step delivery of the food of your choice 40 responses

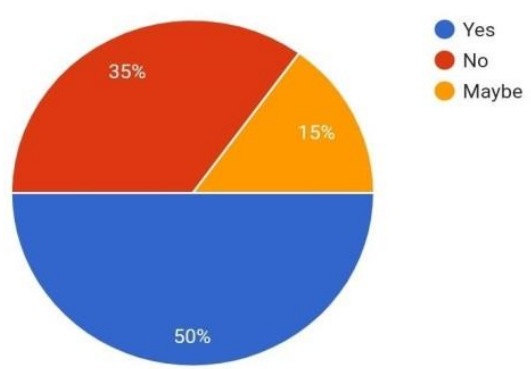


INFERENCE: With the help of fig 7 we can infer that $50 \%$ of the participants admit that they would find it difficult to survive without digitalised food ordering and delivery in the fast paced world.

\section{FINDINGS}

- We can see that majority of the participants actively choose and use digital ordering methods to acquire food rather than use other alternatives like eating out or cooking at home.

- Online Ordering has led to the over indulgence of consumers thereby increasing sales in the fast food business.

Due to the digitalisation of the fast food industry, consumer preferences has favourable changed towards fast food businesses, leading to the boom in fast food industry.

\section{SUGGESTIONS}

- Though digitalisation has affected favourably to fast food businesses, it is important to create consumer awareness with regard to health \& hygiene issues. Consumers need to be aware of the drastic effects of over indulgence while not being prey to the aggressive marketing strategies of the online delivery applications.

- Digitalisation in the fast food industry has led to cut throat competition among the businesses. Quality and hygiene should not be compromised while cutting costs or giving discounts, for the sake of competition.

\section{CONCLUSION}

With reference to the analysis of the above mentioned information, we can see that digitalisation has brought across a revolution in the fast food industry where the power of a click has appeased the consumer's to the extent of drastic increase in sales turnover in these fast food industries.

While this boom is good for the Indian economy, it is the responsibilities of these digital platforms to keep the customers aware of their eating-habits and be ethical about quality and hygiene matters. It is also the responsibility of the consumers to be aware of their indulgence and use their purchasing power wisely.

\section{CITATIONS}

•http://www.citrix.com/Transformation

• http://Ad·www.tetrapak.com/

- Master data collected through primary survey. 